\title{
Links between the dynamical evolution and the surface color of the Centaurs
}

\author{
M. D. Melita ${ }^{1,2}$ and J. Licandro ${ }^{3,4}$ \\ ${ }^{1}$ IAFE (CONICET-UBA), Argentina \\ e-mail: melita@iafe.uba.ar \\ 2 FCEyN (UBA), Argentina \\ 3 Instituto de Astrofísica de Canarias, c/Vía Láctea s/n, 38200 La Laguna, Tenerife, Spain \\ e-mail: jlicandr@iac.es \\ 4 Departamento de Astrofísica, Universidad de La Laguna, 38205 La Laguna, Tenerife, Spain
}

Received 7 June 2011 / Accepted 1 February 2012

\begin{abstract}
Context. The Centaurs are a transitional population of minor bodies of the solar system and the evolutionary link between the transNeptunian objects and the short period comets. The surface properties of these objects are very peculiar, because currently available data suggest that their visual surface colors divide the population into two distinctive groups, those with reddish slopes of the visual reflection spectra and those with neutral spectra. Moreover, some of them are known to posses comas produced by cometary activity. Aims. We aim to investigate possible links between the orbital dynamical history and the surface physical properties of the bodies of this population.

Methods. By means of numerical integrations of the equations of motion we calculated the orbital evolution of three groups of Centaurs: the Red group, the Gray group, and the Active group. We looked for statistical differences in the timescales spent by the objects of each group at heliocentric distances below certain values that are associated with locations where certain particular physical processes occur at the surfaces.

Results. We find remarkable differences when we compare the fraction of objects that penetrate below typical heliocentric distances for each group.

Conclusions. Our results suggest that the observed bimodality in the distribution of surface colors of the Centaurs is caused by the different thermal reprocessing on the surface of bodies of the Red group on one side and the Active and Gray groups on the other. Centaurs of the Gray group likely had cometary activity, therefore their color distribution is similar to that of comet nuclei.
\end{abstract}

Key words. Kuiper belt: general - minor planets, asteroids: general

\section{Introduction}

The Centaurs are a dynamical class of minor bodies of the solar system, short-time residents in the region between Jupiter's and Neptune's orbits, with very chaotic and unstable orbits. They are considered to be transitional bodies from the trans-Neptunian belt (TNB) beyond Neptune to the active Jupiter family comets (JFCs). Following Jewitt (2009), a Centaur is a body with an orbit that meets the conditions $a_{\mathrm{J}}<a<a_{\mathrm{N}}$ and $a_{\mathrm{J}}<q<a_{\mathrm{N}}$, i.e., its perihelion distance $q$ and semimajor axis $a$ lie within the semimajor axis of Jupiter $\left(a_{\mathrm{J}}=5.2 \mathrm{AU}\right)$ and Neptune $\left(a_{\mathrm{N}}=\right.$ $30 \mathrm{AU}$ ). With orbital lifetimes of less than $10^{7}$ years (Holman \& Wisdom 1987), the Centaurs are brief residents in the region between the giant planets. A fraction of those that survive in this region may become JFCs (Levison \& Duncan 1997; Morbidelli 2008).

Centaurs are an intermediate population, halfway between the distant, cold, and relatively stable bodies in the TNB and the rapidly sublimating comets of the hot inner regions. The study of their physical properties and in particular the search for activity among the group is of scientific interest for assessing the relationship between classes of objects and for better understanding the evolution of minor bodies in the solar system.

Remarkably, some Centaurs ( 15 objects of a group of $\sim 150$ members) show comet-like activity even when they are far beyond the orbit of Jupiter, which suggests mass loss driven by a process, which is different from the sublimation of water ice. Many active Centaurs have dust loss rates comparable to, or even higher than, several active comets at much shorter heliocentric distance: for example, P/2004 A1 (LONEOS) has a dust production rate around $100-200 \mathrm{~kg} \mathrm{~s}^{-1}$ up to $6.5 \mathrm{AU}$ (Mazzotta Epifani et al. 2006).

The activity among Centaurs is part of a wider debate on the activity of minor bodies at great distance from the Sun, outside the so-called water zone. Beyond 5 to $6 \mathrm{AU}$, comet-like activity cannot be explained anymore with classical water ice direct sublimation (Meech \& Svoren 2005). Models of cometary nuclei (e.g., Prialnik 1997; Capria et al. 2000a,b) and laboratory experiments (e.g. Notesco et al. 2003) show that amorphous water ice is highly porous and can contain a large number of (hyper)volatile molecules trapped at time of condensation. The trapped gas could be released upon ice crystallization, which would cause dust loss at temperatures too low (and hence at heliocentric distances too far) for crystalline water ice to sublimate. For example, the activity observed in Centaur Chiron has been modeled as a product of crystallization heating by Prialnik et al. (1995).

Considered as a group, the Active Centaurs have smaller perihelia than the population of Centaurs considered as a whole (median 5.73 AU for the Active versus 12.73 AU for all). 
Jewitt (2009) showed that under some hypothesis on surface temperature ranges inside the solar system, the critical distance $\left(R_{\mathrm{C}}\right)$ inside which exposed ice must be crystalline and the phase transition is expected, is $6.8 \leq R_{\mathrm{C}} \leq 14.0 \mathrm{AU}$. Hence, Active Centaurs all have perihelia small enough for the crystallization of amorphous water ice to be a contributing factor to their dust loss.

Centaur objects also exhibit a physical property not observed among any other objects in the solar system, their visual colors divide the population into two distinct groups: Gray and red (Peixinho et al. 2003; Tegler et al. 2003, 2008). Centaurs and trans-Neptunian objects (TNOs) should exhibit a range of surface colors because the solar and cosmic radiation should redden the surfaces, and impacts should puncture the radiationreddened crusts and expose interior, pristine, gray ices (Luu \& Jewitt 1996). Comparing with the $B-R$ color distribution of other population of icy objects, Tegler et al. (2008) concluded that only comet nuclei overlap the Gray Centaur group, and that the color distribution of classic TNOs is very similar to that of the Red Centaur group. In addition, Gray and Red Centaurs exhibit a statistically significant difference in albedo, with the Gray Centaurs having a lower median albedo than the Red Centaurs (Stansberry et al. 2008).

The existence of two color groups of Centaurs may be due to a primordial, temperature-induced, composition gradient, or to the existence of different recent evolutionary processes between the members of the Gray and Red populations (e.g. Tegler et al. 2008; Jewitt 2009). In the first case, most members of the Gray populations would have been formed closer to the Sun, at temperatures high enough for them not to acrete as many carbon-rich volatiles as the Red ones, which are formed at lower temperatures. The hypothesis of different evolutionary processes suggests that Gray Centaurs recently attained heliocentric distances close enough to the Sun to develop dust mantles through cometary-like activity, while the Red ones did not and are covered by organic-rich materials formed through cosmic irradiation.

Most Active Centaurs are slightly redder than the Sun at optical wavelengths, but they appear devoid of the ultrared matter found in the inactive Centaurs and TNOs.

Exploring the hypothesis of a primordial compositional difference between Gray and Red Centaurs is very difficult because of the hightly chaotic nature of their orbits and the asssociated impossibility of deriving from their present orbits where the objects formed. In this project we investigate the existence of different evolutionary processes between the members of the two populations by investigating possible links between the orbital dynamical history and the color of the surface of Centaurs. To this aim, we studied the orbital evolution of three groups: the Red group, the Gray group and the Active group. In particular, we explored whether Centaurs of the Gray group are more likely to have moved closer to the Sun than the Red ones and are therefore more likely to have had cometary activity. Jewitt (2009) showed that most Active Centaurs have colors similar to those of the Gray group, but physical interpretation of the colors, is hampered by coma contamination in the Active group. But there are no significant differences between the orbital elements of the two color groups, either statistically or in absolute magnitude (Tegler et al. 2008). In Sect. 2 we describe the experiment and our dynamical methods. In Sect. 3 we present the results of our search for statistical differences in the timescales spent by the objects of each group at heliocentric distances below which the activity may occur. In Sect. 4 we present our conclusions.

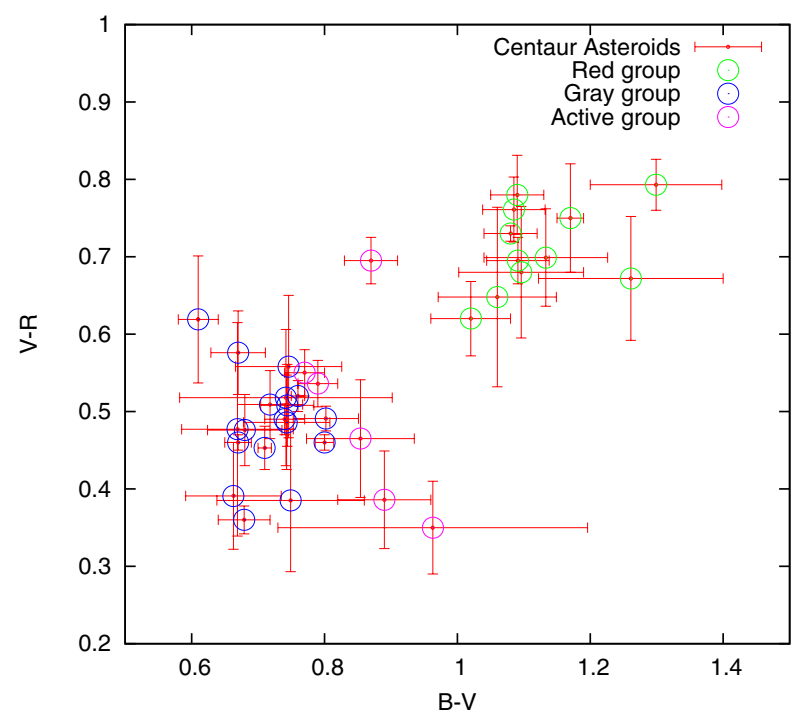

Fig. 1. $V-R$ vs. $B-V$ plot of the Centaurs. Members of each group are indicated.

\section{Experimental setup}

Our goal is to investigate possible correlations between the color indexes of the Centaurs and the tendency of their orbits to approach the inner solar system. Our experiments were set up in the following way: from the set of Centaur objects with known surface color indexes (Hainaut \& Desanti 2002) we divided the sampe into two groups, the so-called Red group $(R)$, and Gray group $(G)$. The color indexes of these two groups satisfy the following criteria:

$$
(B-V)_{R}<1.0
$$

and

$(B-V)_{G}>1.0$,

where $(B-V)>_{R}$ and $(B-V)_{G}$ are the $B-V$ color indexes of the members of the red group and gray group, respectively. The particular values (Hainaut \& Delsanti 2002) are listed in Table 1. The mean values and standard deviations of the $(B-V)$ colors of each group are given in Table 2 . The difference between the means is 0.43 , which is $8.6 \times \sigma_{G}$ and $6.1 \times \sigma_{R}$. But the range of colors of the Centaur asteroids is similar to the other populations of the outer solar system, which range approximately from 0.4 to 1.4. Therefore, we have grounds to believe that there is a physical cause for the grouping.

We also studied the orbital evolution of a group of Active Centaurs (the $A$ group). The spectral properties of these objects are listed in Table 3. In Fig. 1 we plot the color indexes of the Centaurs of the three groups.

\subsection{Methods}

We generated 100 clones of the orbits of each object by producing uniform random variations about their nominal values with a maximum magnitude of $10^{-3} \mathrm{AU}$ on the semimajor axes, $10^{-3}$ on the eccentricities, and $10^{-2}$ degrees on the inclinations. All osculating orbital elements were obtained from the JPL Horizons website (http://ssd.jpl .nasa.gov/?ephemerides) corresponding to Julian date 2454 101.5. Each clone was treated as a massless particle and integrated both in the future and in the 
Table 1. Individual members of the red $(R)$ and gray $(G)$ groups.

\begin{tabular}{lccccc}
\hline \hline Group & Designation & $B-V$ & $\delta(B-V)$ & $V-R$ & $\delta(V-R)$ \\
\hline$G$ & 2000 FZ53 & 0.610 & 0.030 & 0.619 & 0.082 \\
$G$ & 2002 DH5 & 0.663 & 0.072 & 0.391 & 0.069 \\
$G$ & (10370) Hylonome & 0.669 & 0.084 & 0.477 & 0.138 \\
$G$ & (95626) 2002 GZ32 & 0.670 & 0.041 & 0.576 & 0.054 \\
$G$ & (119315) 2001 SQ73 & 0.670 & 0.020 & 0.460 & 0.010 \\
$G$ & (2060) Chiron & 0.679 & 0.039 & 0.360 & 0.018 \\
$G$ & (54598) Bienor & 0.680 & 0.056 & 0.476 & 0.046 \\
$G$ & (32532) Thereus & 0.710 & 0.010 & 0.453 & 0.028 \\
$G$ & (63252) 2001 BL41 & 0.718 & 0.049 & 0.509 & 0.044 \\
$G$ & (120061) 2003 CO1 & 0.740 & 0.030 & 0.490 & 0.020 \\
$G$ & (52872) Okyrhoe & 0.743 & 0.065 & 0.486 & 0.061 \\
$G$ & (60558) Echeclus & 0.742 & 0.160 & 0.518 & 0.088 \\
$G$ & (8405) Asbolus & 0.744 & 0.040 & 0.508 & 0.053 \\
$G$ & (49036) Pelion & 0.746 & 0.080 & 0.558 & 0.092 \\
$G$ & (87555) 2000 QB243 & 0.749 & 0.111 & 0.385 & 0.092 \\
$G$ & (73480) 2002 PN34 & 0.760 & 0.010 & 0.520 & 0.020 \\
$G$ & 2008YB3 & 0.800 & 0.014 & 0.460 & 0.010 \\
$G$ & (10199) Charylko & 0.802 & 0.049 & 0.491 & 0.016 \\
$R$ & (31824) Elatus & 1.020 & 0.060 & 0.620 & 0.048 \\
$R$ & (121725) 1999 XX143 & 1.060 & 0.089 & 0.648 & 0.116 \\
$R$ & (88269) 2001 KF77 & 1.080 & 0.040 & 0.730 & 0.010 \\
$R$ & (83982) 2002 GO9 & 1.085 & 0.047 & 0.761 & 0.042 \\
$R$ & (7066) Nessus & 1.090 & 0.040 & 0.780 & 0.051 \\
$R$ & (55576) Amycus & 1.091 & 0.047 & 0.695 & 0.030 \\
$R$ & (52975) Cyllarus & 1.096 & 0.094 & 0.680 & 0.085 \\
$R$ & (44594) 1999 OX3 & 1.133 & 0.093 & 0.699 & 0.063 \\
$R$ & (111444) 2001 XZ255 & 1.170 & 0.020 & 0.750 & 0.070 \\
$R$ & 1994 TA & 1.261 & 0.139 & 0.672 & 0.080 \\
$R$ & (5145) Pholus & 1.299 & 0.099 & 0.793 & 0.033 \\
\hline & & & & & \\
& & & &
\end{tabular}

past for $10^{6} \mathrm{yr}$ in the gravitational field of the Sun, the four giant planets and the Earth. The numerical integrator scheme used (Brunini \& Melita 2002) is a hybrid symplectic secondorder method. We chose this integration method because it provides fast and reliable results. The integration time step of the symplectic method used was $0.1 \mathrm{yr}$, which is $1 / 10$ of the mean period of the innermost planet considered, and approximately $1 / 100$ of that of Jupiter. A close encounter with a planet is defined when the planetocentric distance is shorter than three Hillradii. The orbit during a close encounter is the one calculated using a Burlish and Stoer integrator with the strategy developed by Chambers (1999), which eliminates the need of changing the Hamiltonian during the event.

\section{Results}

The lifetime of the Centaurs is about $10^{6}$ years (Holman \& Wisdom 1987). On this timescale, we looked for differences in the residence times at low perihelia of orbits belonging to objects of the different groups as defined in Sect. 2. We find that the residence time at low perihelia is significantly longer for the Gray and the Active groups than the one of the Red group (see Fig. 2). Note that time spent at perihelia below $\approx 5 \mathrm{AU}$ is much shorter for the Red group than in the other two groups.

We also computed the fraction, $f$, of objects of each group with perihelia that reach values below certain critical distances, $R_{\mathrm{C} 1}=5 \mathrm{AU}, R_{\mathrm{C} 2}=9.5 \mathrm{AU}$ and $R_{\mathrm{C} 3}=19 \mathrm{AU}$, as a function of time. The fraction $f$ is computed with respect to the total number of objects in each group that remain in the integration, i.e. within semimajor axes shorter than $2000 \mathrm{AU}$, all objects reaching these orbits are assumed to be lost through the interaction with perturbers outside to the solar system. The values of $f$ for
Table 2. Mean color indexes and their corresponding dispersions for the sample taken from MBOSS for the Red $(R)$ and $\operatorname{Gray}(G)$ groups.

\begin{tabular}{lcc}
\hline \hline Group & $\langle B-V\rangle$ & $\sigma$ \\
\hline$G$ & 0.71 & 0.05 \\
$R$ & 1.14 & 0.07 \\
\hline
\end{tabular}

each group, corresponding to integrations in the past and in the future, are shown in Figs. 3 and 4, respectively. Evidently, that the sample of orbits of the Red group have a significantly smaller tendency to penetrate into the inner solar system than those of the Active and the Gray groups, particularly between $5 \mathrm{AU}$ and 9.5 AU over a timescale of about $750.00 \mathrm{yr}$ both in the future and in the past.

\subsection{Are the results uniform across the sample?}

We checked if the tendency to penetrate less into the solar system as detected for the Red group in the previous section, depends on the particular choice of the samples. We investigated if this result is only a selection effect and only depends on the peculiar properties of a few objects, probably less than three, given that there are about ten objects per group. Therefore we devised a "jackknife" strategy to investigate if the differences found between the Red and the Gray groups persist even if three objects are randomly excluded from each group.

This test was performed in the following way. We generated all the possible subsamples, such that each group had three objects less than originally listed in Tables 1 and 3. These ammount to 188100 cases, each with a different "reduced" Red and "reduced" Gray group. For each of these we computed 
Table 3. Individual members of the Active group. The indexes $B-V$ and $V-R$ and the corresponding error values, $\delta(B-V)$ and $\delta(B-V)$ are from the MBOSS database.

\begin{tabular}{lccccc}
\hline \hline Group & Designation & $B-V$ & $\delta(B-V)$ & $V-R$ & $\delta(V-R)$ \\
\hline A & 166P/NEAT & 0.870 & 0.040 & 0.695 & 0.030 \\
A & 167P/CINEOS & 0.770 & 0.030 & 0.550 & 0.030 \\
A & NEAT C/2001 M10 & 0.963 & 0.233 & 0.350 & 0.060 \\
A & (60558) Echeclus & 0.854 & 0.081 & 0.465 & 0.076 \\
A & 39P/Oterma & 0.890 & 0.070 & 0.386 & 0.063 \\
A & 29P/Schwassmann-Wachmann 1 & 0.790 & 0.030 & 0.536 & 0.030 \\
\hline
\end{tabular}
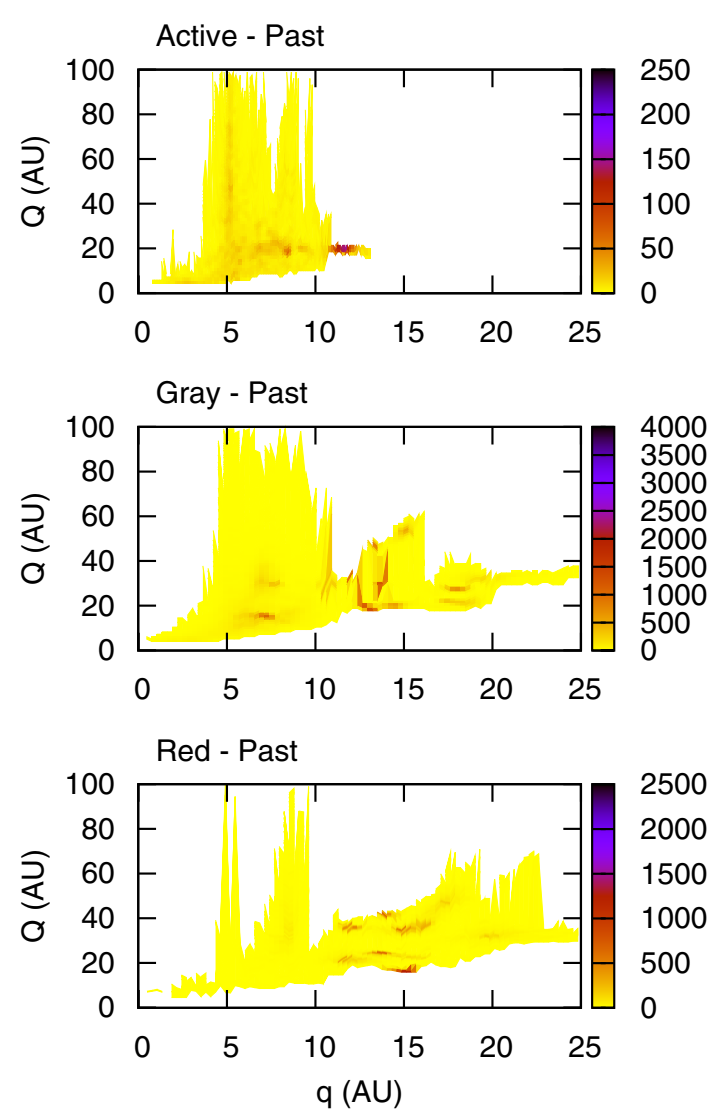


Fig. 2. Residence maps. The colorscale is proportional to the time spent at the indicated aphelion (Q) and perihelion (q) distances.

the time-averaged fraction of orbits that reach perihelia below $R_{\mathrm{C} 2}=9.5 \mathrm{AU},\langle N(q<9.5 \mathrm{AU}) / N T\rangle$, both for the Red and the Gray reduced groups. The averages were derived over an integration time of only $10^{5} \mathrm{yr}$, because performing the calculation over a longer timescale makes a prohibitive computing time.

We verified that in all cases, the values corresponding to the Red reduced groups are lower than those of the Gray reduced groups (see Fig. 5). Clearly the results for the Red group are consistently more robust. For the Gray group, the tendency to penetrate into the inner solar system - at least below $9.5 \mathrm{AU}-$ is not so uniform across the sample and there are a few objects that dominate in the overall statistics. These particluar objects are (2060) Chiron, (32532) Thereus, (52872) Okhyroe, and (63252) 2001 BL41. We conclude that the Red group has a consistently lower tendency to penetrate below 9.5 AU, and the tendency in the Gray group is more widespread.

\section{Conclusions}

Two different scenarios were proposed to explain the existence of two color groups of Centaurs (e.g. Tegler et al. 2008; Jewitt 2009): (1) the existence of different evolutionary processes; (2) a primordial, temperature-induced composition gradient. We investigated the existence of different evolutionary processes between the members of the two populations.

According to a scenario in which activity is driven by trapped gasses in amorphous ice, there are typical temperatures at which particular gas-molecules are released from the amorphous icy matrix. Therefore, in the environment of the Sun as a mainsequence star, some correlation between distances and surface properties was suggested (Bar Nun et al. 1987). If the dominant phenomenon that modifies the surface is cometary-like activity, those properties must be related to the time spent at lowperihelion distances, i.e. below the distance corresponding to the critical distance $\left(R_{\mathrm{C}}\right)$ inside which the water-ice amorphouscrystalline phase transition occurs $\left(6.8 \leq R_{\mathrm{C}} \leq 14.0 \mathrm{AU}\right)$, which allows the release of the typical volatiles trapped in the water ice.

We measured the number of orbits associated with each group of Centaur asteroids that spend time below three typical distances $R_{\mathrm{C} 1}=5 \mathrm{AU}, R_{\mathrm{C} 2}=9.5 \mathrm{AU}$, and $R_{\mathrm{C} 3}=19 \mathrm{AU}$. We found an apparent tendency for the orbits of objects belonging to the Red group to penetrate less into the solar system than those 

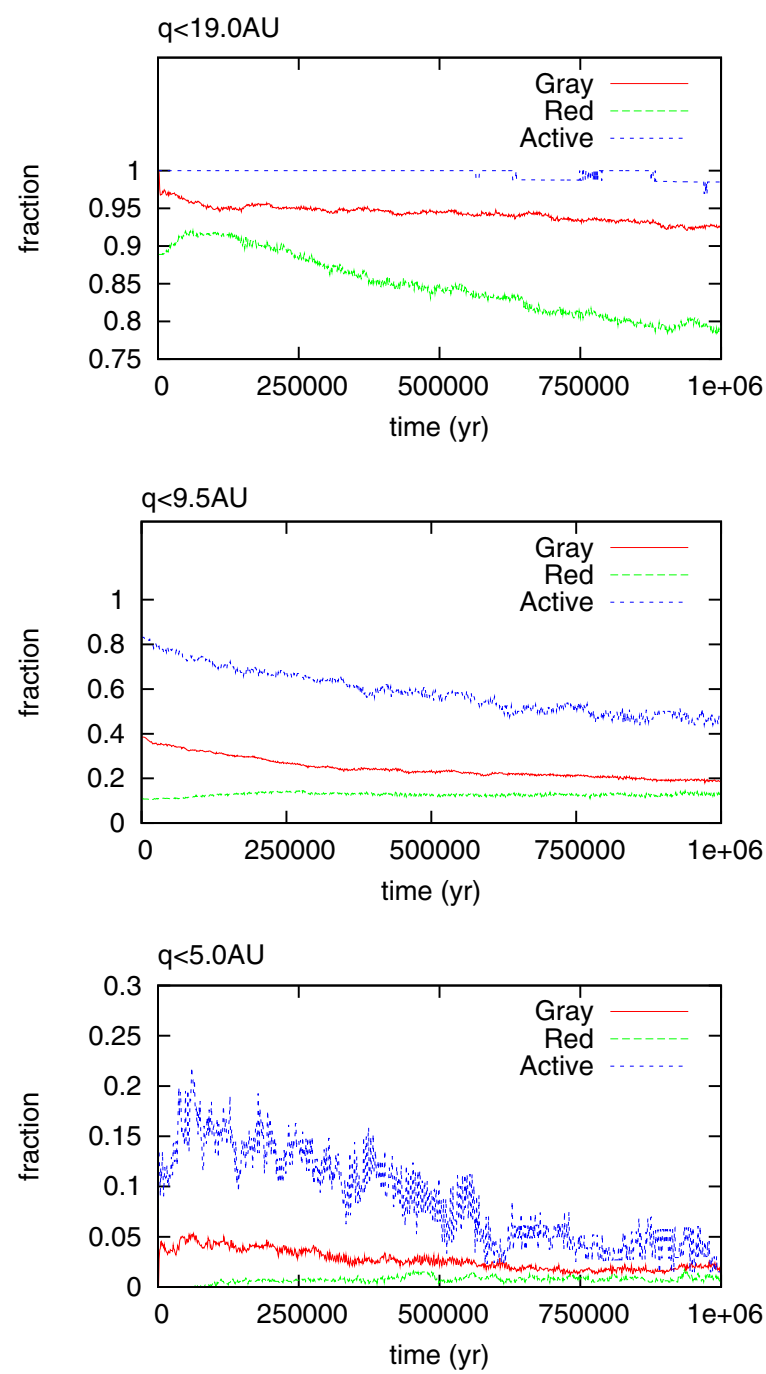

Fig. 3. Fraction, $f$, of clones with perihelia that reach values lower than indicated. Numerical integrations in the future.

of the Gray group and the Active group, respectively. The difference is most pronounced when we consider $R_{\mathrm{C} 2}=9.5 \mathrm{AU}$, which suggests that the phenomenon causing the difference is the release of trapped gasses in amorphous ice.

The tendency shown between Red and Gray Centaurs was confirmed when we consider the Active Centaurs, which, as a group, show a much higher probability to have spent time at short distances from the Sun.

In conclusion, our results suggest that the observed bimodality in the distribution of surface colors of the Centaurs is likely caused by the different thermal processing suffered on the surface of bodies of the Red and Gray groups. Centaurs of the Gray group likely had experienced cometary activity driven by the release of volatiles in the past, and as a result of it, their surfaces are covered by a cometary-like dust mantle (Rickman 1990) and thus have a surface-color distribution similar to that of Active Centaurs, comets, and cometary nucleii.

Acknowledgements. We are grateful to Julio Fernández for his useful comments and discussions. J. L. gratefully acknowledges support from the Spanish "Ministerio de Ciencia e Innovación" project AYA2010-19776. M. D. M. acknowledges support from ANPCyT grant PICT 0218/2007 and CONICET grant PIP 11220090100461/2010.
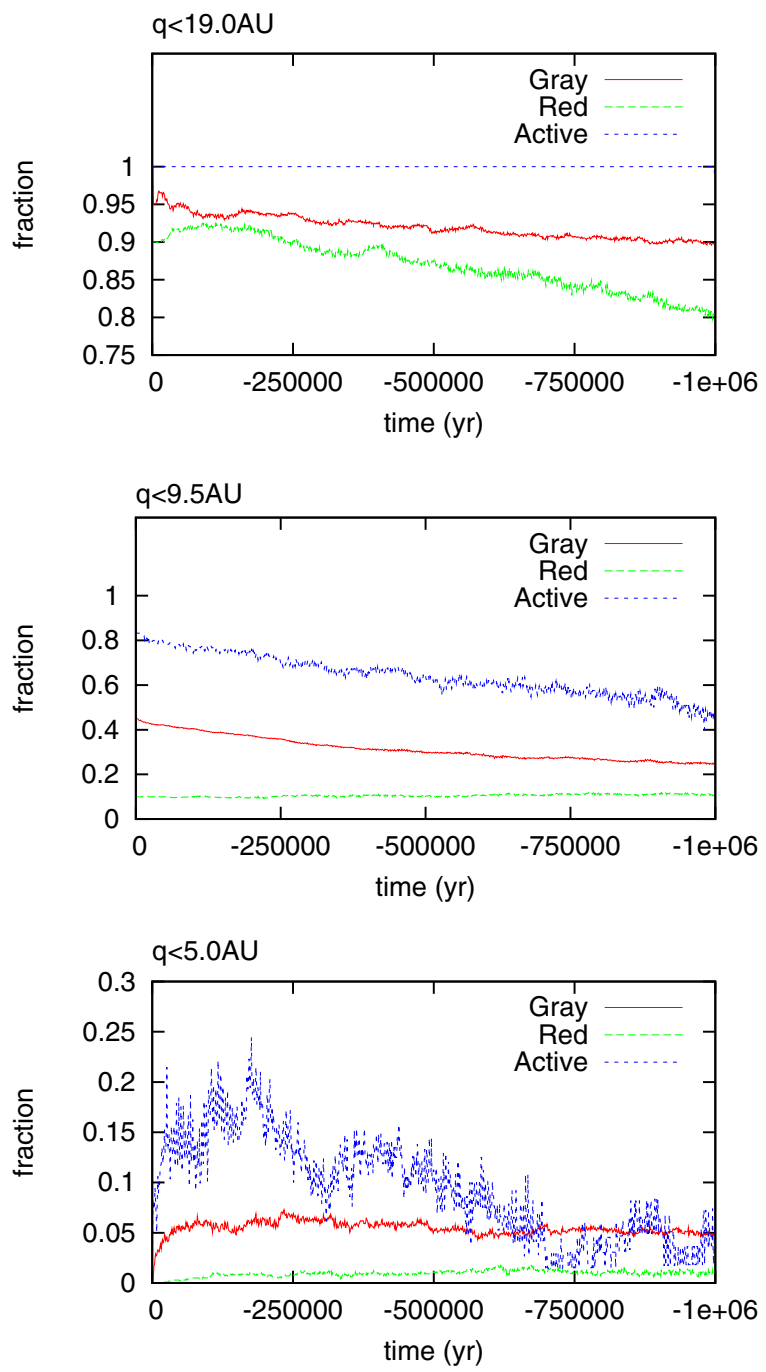

Fig. 4. Fraction, $f$, of clones with perihelia that reach values lower than indicated. Numerical integrations in the past.

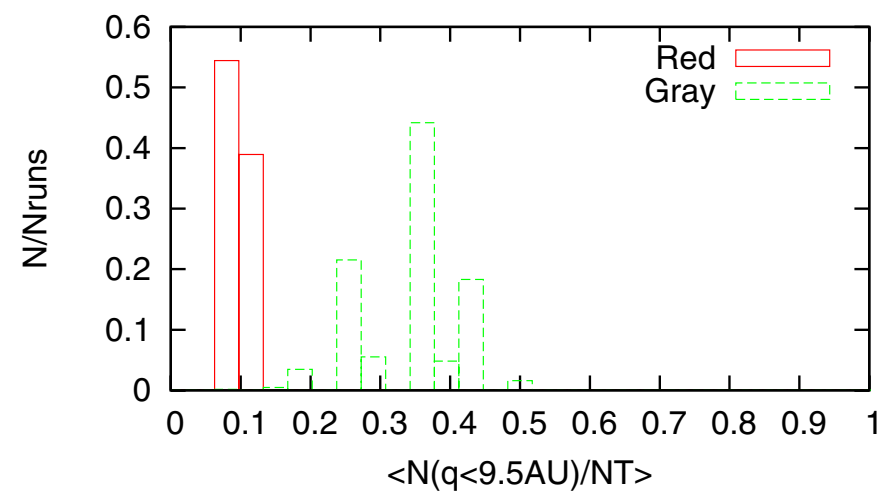

Fig. 5. Histogram of the fraction of cases (of a total of 188100 randomly generated cases) as a function of the the value of the time-averaged fraction of orbits that reach perihelia below $R_{\mathrm{C} 2}=9.5 \mathrm{AU},\langle N(q<$ $9.5 \mathrm{AU}) / N T\rangle$.

\section{References}

Bar-Nun, A., Dror, J., Kochavi, E., \& Laufer, D. 1987, Phys. Rev. B, 35, 2427 Brunini, A., \& Melita, M. D. 2002, Icarus, 160, 32 
A\&A 539, A144 (2012)

Capria M. T., Coradini, A., De Sanctis, M. C., \& Orosei, R. 2000a, A\&A, 357, 359

Capria, M. T., Coradini, A., De Sanctis, M. C., \& Orosei, R. 2000b, AJ, 119, 3112

Chambers, J. E. 1999, MNRAS, 304, 793

Hainaut, O., \& Delsanti, A. 2002, A\&A, 389, 641

Holman, M. J., \& Wisdom, J. 1993, AJ, 105, 1987

Jewitt, D. 2009, ApJ, 137, 4296

Levison, H. F., \& Duncan, M. J. 1997, Icarus, 127, 13

Luu, J., \& Jewitt, D. 1996, AJ, 112, 2310

Mazzotta, Epifani, E., Palumbo, P., Capria, M. T., et al. 2006, A\&A, 460, 935

Meech, K. J., \& Svoren, J. 2005, in Comets I I (Tucson: Univ. Arizona Press)

Morbidelli, A. 2008, Saas-Fee Advanced Course 35: Trans-Neptunian Objects and Comets (Berlin: Springer-Verlag), 79
Notesco, G., Bar-Nun, A., \& Owen, T. 2003, Icarus, 162, 183 Prialnik, D. 1997, ApJ, 478, L107

Prialnik, D., Brosch, N., \& Ianovici, D. 1995, MNRAS, 276, 1148

Peixinho, N., Doressoundiram, A., Delsanti, A., et al. 2003, A\&A, 410, L29

Rickman, H., Fernández, J., \& Gustafson, B. 1990, A\&A, 237, 524

Tegler S. C.,\& Romanishin W. 2003, Icarus, 161, 181

Tegler S. C., Bauer J. M., Romanishin, W., \& Peixinho, N. 2008, in The Solar System Beyond Neptune, ed. M. A. Barucci, H. Boehnhardt, D. P. Cruikshank, \& A. Morbidelli (Tucson: University of Arizona Press), 105

Stansberry, J., Grundy, W., Brown, M., et al. 2008, in The Solar System Beyond Neptune, ed. M. A. Barucci, H. Boehnhardt, D. P. Cruikshank, \& A. Morbidelli (Tucson: University of Arizona Press) 